\title{
CryoEM Analysis of Lecithin:Cholesterol Acyltransferase in Complex with High- Density Lipoprotein Particles
}

\author{
Kelly Manthei ${ }^{1}$, Louise Chang ${ }^{1}$, John Tesmer $^{2}$ and Melanie Ohi ${ }^{1}$ \\ ${ }^{1}$ University of Michigan, Ann Arbor, Michigan, United States, ${ }^{2}$ Purdue University, West Lafayette, \\ Indiana, United States
}

Coronary heart disease (CHD) is an atherosclerotic disease that occurs when plaque builds up in the arterial wall, ultimately leading to heart failure, heart attack, arrhythmias, and angina. It has been long established that there is an inverse relationship between the risk for CHD and the levels of high density lipoprotein (HDL) cholesterol (HDL-C). HDL particles are key players in the process of reverse cholesterol transport, where they move cholesterol from arterial plaques to the liver to be excreted. Central to this process, lecithin:cholesterol acyltransferase (LCAT) catalyzes a critical step wherein it transfers the sn-2 acyl group from phosphatidylcholine to cholesterol within HDL particles. This process promotes maturation of discoidal pre- $\beta$ HDL to spherical HDL and further cholesterol efflux (1). As CHD is the leading cause of death worldwide, efforts to enhance HDL-C levels via LCAT activation are of global interest. Furthermore, treatments could also be beneficial to those with familial LCAT deficiency, which is an LCAT genetic disease ultimately resulting in renal failure. We have focused on structural characterization of the LCAT-HDL complex in order to aid in the development of therapeutics targeting LCAT.

LCAT is activated by apolipoprotein A-I (ApoA-I), the most abundant structural apolipoprotein in HDL $(2,3)$. The two bound molecules of ApoA-I are predicted to form ten tandem amphipathic $\alpha$-helices that wrap in anti-parallel fashion around the lipid bilayer, forming a "double belt" (4). Our previous crystal structures of LCAT indicate that activation might be mediated by displacement of a lid covering the active site of $\operatorname{LCAT}(5,6)$. We recently reported a collaborative effort that used hydrogen-deuterium exchange mass spectrometry, crosslinking, and negative stain electron microscopy to put together a low-resolution model of the complex (7). Our data reveal that two LCAT proteins bind to the outer helical belt of ApoAI and do so predominantly at helix 6, allowing us to propose a model for how LCAT interacts with the HDL via both ApoA-I and lipids.

However, there remains a lack of detailed information regarding the specific interactions and orientation of LCAT when bound to HDL that would help us understand mechanisms of activation and how substrates gain access to the active site. Thus, we have turned to cryo electron microscopy (cryoEM) to characterize the LCAT-HDL complex in vitrified ice. The LCAT-HDL complex was visualized in micrographs collected at $200 \mathrm{keV}$ on Talos Arctica and Glacios cryo transmission electron microscopes (Thermo Fisher) (Figure 1). We have collected multiple datasets of the WT LCAT-HDL complex and of a mutant LCAT that cannot be activated by ApoA-I. The data were initially processed using Warp (8), and the resulting particle stack was classified in cryoSPARC v2 (9). The $2 \mathrm{D}$ class averages are promising in that we observe secondary structure features of LCAT that allow us to dock in our crystal structures of LCAT (Figure 2) (5). Focused refinement in RELION-3 (10) has increased the resolution of the map to $\sim 7 \AA$. However, there are challenges in classifying and aligning the particles, which is limiting the potential of the data. This will be addressed by careful data processing and the addition of recently collected data at a $40^{\circ}$ tilt that we hope will generate a high resolution map (11). 


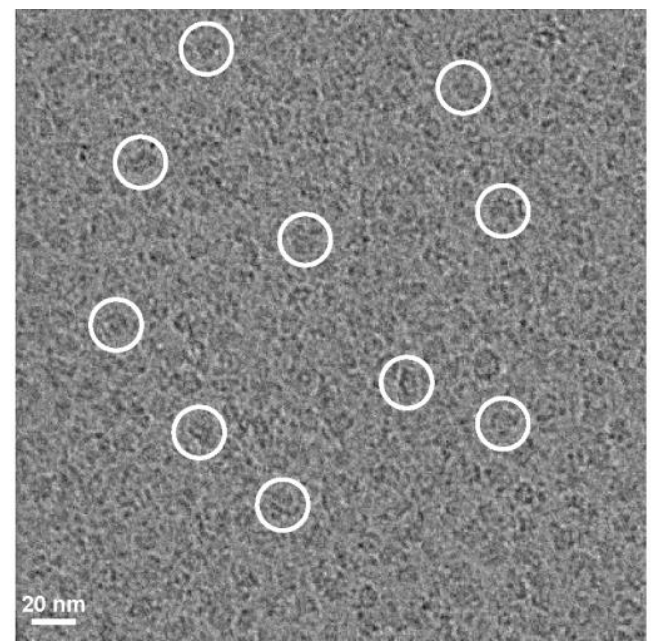

Figure 1. CryoEM micrograph of the LCAT-HDL complex. The micrograph is representative of the LCAT-HDL complex in vitreous ice. Select individual particles have been circled.
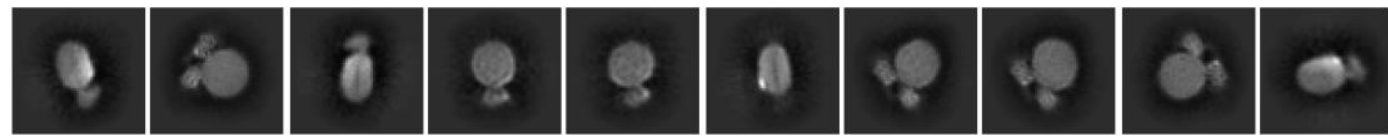

Figure 2. 2D classification of the LCAT-HDL complex. Representative class averages of the complex generated with cryoSPARC. The box size is $\sim 300 \AA$.

References

1. Glomset JA. The plasma lecithins:cholesterol acyltransferase reaction. Journal of Lipid Research. 1968;9(2):155-67.

2. Fielding CJ, Shore VG, Fielding PE. A protein cofactor of lecithin:cholesterol acyltransferase. Biochemical and Biophysical Research Communications. 1972;46(4):1493-8.

3. Jonas A. Lecithin cholesterol acyltransferase. Biochimica et Biophysica Acta. 2000.

4. Sorci-Thomas MG, Bhat S, Thomas MJ. Activation of lecithin:cholesterol acyltransferase by HDL ApoA-I central helices. Clinical lipidology. 2009;4(1):113-24.

5. Manthei KA, Yang SM, Baljinnyam B, Chang L, Glukhova A, Yuan W, et al. Molecular basis for activation of lecithin:cholesterol acyltransferase by a compound that increases HDL cholesterol. Elife. $2018 ; 7$.

6. Manthei KA, Ahn J, Glukhova A, Yuan W, Larkin C, Manett TD, et al. A retractable lid in lecithin:cholesterol acyltransferase provides a structural mechanism for activation by apolipoprotein A-I. Journal of Biological Chemistry. 2017;292(49):20313-27.

7. Manthei KA, Patra D, Wilson CJ, Fawaz MV, Piersimoni L, Shenkar JC, et al. Structural analysis of lecithin:cholesterol acyltransferase bound to high density lipoprotein particles. Commun Biol. 2020;3(1):28.

8. Tegunov D, Cramer P. Real-time cryo-electron microscopy data preprocessing with Warp. Nature methods. 2019:1-7.

9. Punjani A, Rubinstein JL, Fleet DJ, Brubaker MA. cryoSPARC: algorithms for rapid unsupervised cryo-EM structure determination. Nat Methods. 2017;14(3):290-6.

10. Zivanov J, Nakane T, Forsberg BO, Kimanius D, Hagen WJ, Lindahl E, et al. New tools for automated high-resolution cryo-EM structure determination in RELION-3. Elife. 2018;7:e42166.

11. This work was supported by National Institutes of Health (NIH) grants HL131288 to KAM, HL122416 to JJGT and an American Heart Association postdoctoral fellowship 15POST24870001 to KAM. 